\title{
Mechanisms of HIV-tat-Induced Phosphorylation of N-Methyl-D-Aspartate Receptor Subunit 2A in Human Primary Neurons
}

\author{
Implications for NeuroAIDS Pathogenesis
}

\author{
Jessie E. King, ${ }^{*}$ Eliseo A. Eugenin, ${ }^{*}$ \\ Joy E. Hazleton, ${ }^{*}$ Susan Morgello, ${ }^{\dagger}$ \\ and Joan W. Berman* \\ From the Departments of Pathology,* and Microbiology and \\ Immunology, The Albert Einstein College of Medicine, Bronx; \\ and the Department of Pathology (Division of Neuropathology) \\ and Neuroscience, ${ }^{\dagger}$ Mount Sinai Medical Center, School of \\ Medicine, New York, New York
}

HIV infection of the central nervous system results in neurological dysfunction in a large number of individuals. NeuroAIDS is characterized by neuronal injury and loss, yet there is no evidence of HIV-infected neurons. Neuronal damage and dropout must therefore be due to indirect effects of HIV infection of other central nervous system cells through elaboration of inflammatory factors and neurotoxic viral proteins, including the viral transactivator, tat. We previously demonstrated that HIV-tat-induced apoptosis in human primary neurons is dependent on $N$-methyl-Daspartate receptor (NMDAR) activity. NMDAR activity is regulated by various mechanisms including NMDAR phosphorylation, which may lead to neuronal dysfunction and apoptosis in pathological conditions. We now demonstrate that tat treatment of human neurons results in tyrosine $(\mathrm{Y})$ phosphorylation of the NMDAR subunit $2 A$ (NR2A) in a src kinasedependent manner. In vitro kinase assays and in vivo data indicated that NR2A Y1184, Y1325, and Y1425 are phosphorylated. Tat treatment of neuronal cultures enhanced phosphorylation of NR2A Y1325, indicating that this site is tat sensitive. Human brain tissue sections from HIV-infected individuals with encephalitis showed an increased phosphorylation of NR2A Y1325 in neurons as compared with uninfected and HIV-infected individuals without encephalitis. These findings suggest new avenues of treatment for $\mathrm{HIV}$-associated cognitive impairment. (Am J Pathol 2010, 176:2819-2830; DOI: 10.2353/ajpath.2010.090642)

HIV infection causes varying degrees of cognitive impairment in a significant number of individuals. ${ }^{1,2}$ Damage to the central nervous system is a multifactorial process including early viral entry, neuroinflammation, and secretion of toxic factors. ${ }^{3}$ During this process, neuronal damage and apoptosis occur, ${ }^{4}$ but there is little evidence that neurons are infected with HIV. ${ }^{5,6}$ Therefore, the neuronal damage and death characteristic of neuroAIDS must be mediated through indirect mechanisms. ${ }^{3}$ HIV-tat, the transactivator of the virus, is one such toxic factor that causes apoptosis in cultured human neurons. ${ }^{7-11}$

Binding of tat to the low density lipoprotein receptorrelated protein (LRP) on neurons results in the formation of a macromolecular complex at the neuronal cell membrane between LRP and the N-methyl-D-aspartate receptor (NMDAR), mediated by the scaffolding protein PSD95. ${ }^{9}$ In this complex, the NMDAR plays a critical role in the process of tat induced apoptosis in human primary neurons, as blocking with specific NMDAR inhibitors ab-

Supported by NIH Centers for AIDS Research grant (CFAR) AI-051519 at the Albert Einstein College of Medicine. This work was also supported by National Institute of Mental Health grants MH075679 and MH083497 (to J.W.B.), grant MH070297 (to J.W.B. and J.E.K.), and grants R24MH59724 and U01083501 (to S.M.); by a KO1 grant from the National Institute of Mental Health (MH076679 to E.A.E.); by the Medical Scientist Training Program (T32 GM007288 to J.E.K. and J.E.H.); and HIV/AIDS and Opportunistic Infections institutional training grant T32 Al-007501 (to J.E.H.).

Accepted for publication February 25, 2010.

A guest editor acted as editor-in-chief for this manuscript. No person at Thomas Jefferson University or Albert Einstein College of Medicine was involved in the final disposition for this article.

Address reprint requests to Dr. Joan W. Berman, Ph.D., Department of Pathology, F727, The Albert Einstein College of Medicine, 1300 Morris Park Avenue, Bronx, NY 10461. E-mail: joan.berman@einstein.yu.edu. 
rogated cell death completely. ${ }^{8,9}$ The specific mechanisms by which tat alters NMDAR activity are unknown.

NMDAR phosphorylation results in alterations in NMDAR activity, ${ }^{12-14}$ protein-protein interactions, ${ }^{15}$ and trafficking. ${ }^{15-17}$ In pathological conditions, overactivation of the NMDAR results in toxicity. ${ }^{18}$ Studies in rodent models indicate that postsynaptic density proteins facilitate the interaction of kinases, such as src, ${ }^{19}$ fyn, ${ }^{20,21}$ and pyk2 $2^{22-24}$ with the NMDAR, resulting in phosphorylation of its different subunits. As PSD-95 is recruited to the NMDAR after tat treatment, ${ }^{9}$ we examined tat induced association of these kinases with the NMDAR subunit 2A (NR2A), the main subunit present in the macromolecular complex induced by tat, ${ }^{9}$ and subsequent changes in NR2A phosphorylation.

We demonstrate that tat induces tyrosine phosphorylation of the NR2A subunit by a mechanism that involves recruitment of active src to the receptor. We also identify in vitro three tyrosine $(\mathrm{Y})$ residues phosphorylated on human NR2A in a src-dependent manner. One of these, Y1325, showed significantly enhanced phosphorylation in response to tat treatment of human neurons that was dependent on src activity. We also demonstrate in vivo that phosphorylation of $Y 1325$ is significantly increased in neurons present in HIV encephalitic brain tissue sections as compared with those from uninfected and HIV-infected individuals without encephalitis.

\section{Materials and Methods}

\section{Materials}

Neurobasal media, $\mathrm{N}_{2}$ supplement, penicillin/streptomycin (P/S) and trypsin-EDTA were from GibcoBRL (Grand Island, NY). NMDA, glycine, polyclonal antibody to NMDAR1, rabbit polyclonal antibody to MAP-2, antimouse $\mathrm{Cy} 3$, and anti-rabbit fluorescein isothiocyanate secondary antibodies were from Sigma Chemical (St. Louis, MO). Chicken polyclonal antibody to MAP-2 and nonimmune chicken IgY were from Aves Labs (Tigard, OR). Monoclonal antibodies to the NMDAR subunits NR2A, NR2B, and fyn were from Zymed (San Francisco, CA). Polyclonal and monoclonal antibodies to pyk2, as well as normal rabbit IgG, were from Santa Cruz Biotechnology (Santa Cruz, CA). Phosphotyrosine antibody, PTyr-100, src rabbit polyclonal antibody 36D10, and phosphor-specific antibody for active src rabbit polyclonal antibody were from Cell Signaling (Beverly, MA). A second phosphospecific antibody for active src was from Biosource. Control IgG1 and IgG2A purified mouse antibodies were from ICN/Cappel (Salon, OH). Anti-chicken Cy5 secondary antibody was from Abcam (Cambridge, MA). Anti-mouse Alexa Fluor 488 and anti-rabbit Alexa Fluor 594 were from Invitrogen. Src kinase inhibitor I was from Calbiochem (Darmstadt, Germany). Src kinase was purchased from Upstate (Billerica, MA) and EasyTides gamma ${ }^{32}$ P-ATP from Perkin Elmer (Melville, NY). Isostrips for antibody isotyping and complete protease inhibitor tablet were from Roche Diagnostics (Manheim, Germany). NR2A peptides for the in vitro kinase assay were synthesized by GenScript (Piscataway, NJ). Recombinant HIV-1 tat protein (1-72) was from Dr. Avindra Nath, Johns Hopkins Medical Center.

\section{Primary Human Neuronal Cultures}

Human fetal cortical tissue was used as part of an ongoing research protocol approved by the Albert Einstein College of Medicine. Brain tissue was cultured according to previously published protocols. ${ }^{8}$ After 7 to 10 days in culture, the cells were dissociated with trypsin-EDTA and plated onto 100-mm dishes, 35-mm culture dishes, or 24-well tissue culture plates with cover slips in Neurobasal media plus $\mathrm{N}_{2}$ supplement, 0 to $1 \%$ fetal bovine serum, and $1 \%$ Pen/Strep. Media was replaced every 5 days. This resulted in mixed cultures of neurons and astrocytes with no evidence of microglial contamination as determined by immunohistochemical staining for CD68, CD11b, and CD14 (data not shown). To test for neuronal maturation and NMDAR expression, time-lapse calcium imaging was performed to determine responsiveness to NMDA/glycine. Briefly, cells were loaded with $10 \mu \mathrm{mol} / \mathrm{L}$ Fluo-4 AM for 15 to 30 minutes at room temperature. Neurons were imaged in the AECOM Analytical Imaging Facility with a 20X objective using an Olympus IX81 inverted microscope run with OpenLab software. Fields were chosen for the maximal number of neurons, and excitotoxic concentrations of $60 \mu \mathrm{mol} / \mathrm{L}$ NMDA and $10 \mathrm{nmol} / \mathrm{L}$ glycine were added to the culture media to stimulate maximal NMDAR activation. Cultures with 25 to $35 \%$ of neurons responsive to NMDA/glycine treatment were considered mature.

\section{Treatment of Neuronal Cultures}

The exact concentrations of tat in vivo are not known. However, it is likely that tat concentrations could be relatively high in small, localized areas of release. We used tat at a range of 10 to $300 \mathrm{ng} / \mathrm{ml}$, which is equivalent to approximately 1.2 to $35.6 \mathrm{nmol} / \mathrm{L}$, based on the concentration at which each preparation induced apoptosis, chemokine production and/or cellular activation. ${ }^{25-35}$ Lyophilized tat protein was resuspended at $50 \mathrm{ng} / \mu \mathrm{L}$ in $50 \mathrm{mmol} / \mathrm{L}$ Tris buffer containing $100 \mathrm{mmol} / \mathrm{L} \mathrm{NaCl}, 1 \mathrm{mmol} / \mathrm{L} \mathrm{CaCl}_{2}$, and $0.5 \mathrm{mmol} / \mathrm{L}$ dithiothreitol . Concentration of tat treatment was dependent on the activity of the recombinant protein as determined by its ability to induce $>75 \%$ apoptosis in our cultures, and different preparations ranged from 10 to 300 $\mathrm{ng} / \mathrm{ml}$ (equivalent to approximately 1.2 to $35.6 \mathrm{nmol} / \mathrm{L}$ ). This is much lower than concentrations used by others ${ }^{25,32}$ to demonstrate neurotoxicity. These preparations were pure recombinant tat protein, as determined by Coomassie staining, and endotoxin was removed before lyophilization. Control cells were treated with the appropriate amount of vehicle. Previous studies using delta tat (tat with amino acids 48-56 deleted) as a control found no induction of chemokine secretion ${ }^{29}$ or apoptosis (data not shown). ${ }^{32}$ Src kinase inhibitor I (SrCl) was used at $5 \mu \mathrm{mol} / \mathrm{L}$ and added $10 \mathrm{~min}$ utes before tat treatment. All control cultures were treated with the appropriate diluents (vehicle). 


\section{Coimmunoprecipitation}

Following tat treatment, cells were washed twice with cold PBS, harvested in lysis buffer (0.5\% Triton X-100, 50 $\mathrm{mmol} / \mathrm{L}$ HEPES ( $\mathrm{pH} 7.4), 40 \mathrm{mmol} / \mathrm{L}$ sodium chloride, 2 $\mathrm{mmol} / \mathrm{L}$ EDTA, $1.5 \mathrm{mmol} / \mathrm{L}$ sodium vanadate, $50 \mathrm{mmol} / \mathrm{L}$ sodium fluoride, $10 \mathrm{mmol} / \mathrm{L}$ sodium pyrophosphate, and $10 \mathrm{mmol} / \mathrm{L}$ sodium $\beta$-glycerolphosphate) or in radioimmunoprecipitation assay buffer with phosphatase inhibitors (Cell Signaling, Danvers, MA), with protease inhibitors (Roche, Manheim, Germany) added and sonicated for 10 seconds. The lysate was then centrifuged for 5 minutes to remove particulate matter. The amount of protein obtained was quantified by Bradford assay (Bio-Rad, Hercules, CA). Before the addition of specific antibodies, 150 to $250 \mu \mathrm{g}$ of protein were washed and pre-cleared with Immunopure immobilized protein G (Pierce, Rockford, $I L)$. The supernatant was incubated with the appropriate antibody (NR2A, 2.5-3.5 $\mu \mathrm{g})$ or control antibody $\left(\mathrm{IgG}_{1}\right.$, $3.5 \mu \mathrm{g}$ ) overnight at $4^{\circ} \mathrm{C}$, followed by $10 \mu$ l of protein $\mathrm{G}$ for 1 to 3 hours and pelleted. The pellets were washed three times with lysis buffer, resuspended in sample buffer, separated by SDS-polyacrylamide gel electrophoresis and electrotransferred to nitrocellulose or PVDF for analysis by Western blotting.

\section{Western Blot Analyses}

Lysate was prepared as for immunoprecipitation. Protein concentration was determined by the Bradford method. Protein, $60-100 \mu \mathrm{g}$, was resolved in $10 \%$ or $12 \%$ SDSpolyacrylamide gel electrophoresis gels (Bio-Rad) or NuPage gels (Invitrogen) and then electrotransferred to nitrocellulose or PVDF. Nonspecific protein binding was blocked with 5\% nonfat milk in Tween (TBS-T) for 1 hour, followed by overnight incubation at $4^{\circ} \mathrm{C}$ with primary antibodies at the following dilutions: anti-NR2A (1:250), antipTyr-100 (1:2000) anti-pY416 src (Cell Signaling, 1:1000, Biosource, 1:500), anti-src (1:1000), anti-pyk2 (1:1000), anti-fyn (1:500), anti-Y1184 sera (1:100), anti-Y1325 sera (1:100), anti-Y1423 sera (1:100), and anti-pY1325 hybridoma supernatant (1:500-1000). The membrane was then washed with TBS-T and incubated with anti-mouse (1:5000) or anti-rabbit (1:2000) IgG antibody conjugated to horseradish peroxidase. Antigen-antibody complexes were detected on film using ECL reagent (Perkin-Elmer, Waltham, MA) or SuperSignal West Femto Chemiluminescent substrate (Pierce/Thermo Scientific, Rockford, IL).

\section{In Vitro Kinase Phosphorylation Assay}

To identify potential tyrosine phosphorylation sites in human NR2A, the amino acid sequence of NR2A (NCBI accession no. NP_000824) was input into NetPhos 2.0. ${ }^{36}$ Peptides containing those tyrosines in the cytoplasmic tail that scored $>0.8$ or had been referenced in the literature as potential phosphorylation sites were synthesized and used in our in vitro kinase assay. Peptides were synthesized by the Genscript Corporation and had the following sequences: src target consensus se- quence (EEVYQQWSQ ${ }^{37}$ ), Y1151 (FPDPYQDPS), Y1184 (NNDQYKLYS), Y1292 (RQHSYDNIV), Y1325 (EGNFYGSLF), Y1387 (PSPPYKHSL), Y1400 (VNDSYLRSS), and Y1423 (HNDVYISEH). Src in vitro kinase assays were performed by adding 3 active units of recombinant src or distilled, deionized water to a reaction solution containing 20 $\mathrm{mmol} / \mathrm{L}$ HEPES, $\mathrm{pH} 7.4,5 \mathrm{mmol} / \mathrm{L} \mathrm{MgCl}_{2}, 5 \mathrm{mmol} / \mathrm{L}$ $\mathrm{MnCl}_{2}, 25 \mu \mathrm{mol} / \mathrm{L}$ cold ATP, and $1 \mu \mathrm{Ci} \gamma^{32}$ P-ATP. After 10 minutes incubation at room temperature, $2 \mu \mathrm{g}$ of the consensus sequence peptide or NR2A peptides were added to each reaction and were incubated for $45 \mathrm{~min}$ utes at $37^{\circ} \mathrm{C}$. To stop the reaction, $2.2 \mu \mathrm{l}$ of $5 \mathrm{X}$ protein buffer was added and was incubated for 5 minutes at $90^{\circ} \mathrm{C}$. Peptides were then run on an Invitrogen $12 \%$ BisTris gel with $1 X$ MES buffer, $130 \mathrm{~V}$ for 40 to 50 minutes. Gels were imaged using Fuji RX X-ray film.

\section{Generation of Phospho Site-Specific Monoclonal Antibodies}

Peptides containing phosphorylated tyrosines were synthesized by Genscript with the following sequences: pY1184 (KNNDQ\{pTYR\}KLYS), pY1325 (KEGNF\{pTYR\} GSLF), and pY1423 (KHNDV\{PTYR\}ISEH) and coupled to keyhole limpet hemocyanin using the Imject Immunogen EDC kit with mcKLH and OVA (Pierce) as per the manufacturer's instructions. Four 6- to 8-week-old female $\mathrm{BALB} / \mathrm{c}$ mice were immunized for each antigen with AdjuPrime immune modulator (Pierce) and keyhole limpet hemocyanin-phosphopeptide every two weeks for 6 weeks, and titers were assayed at week 7 . Mice were subsequently boosted and sera were redrawn two weeks later and assayed by enzyme-linked immunosorbent assay for reactivity to keyhole limpet hemocyanin, unphosphorylated peptide, and phosphorylated peptide. The sera were pre-absorbed against the unphosphorylated forms of the peptides, and tested and used for Western blotting analysis. Based on data with the anti-sera, the mouse with the best phospho-specific response to Y1325 was used to perform the spleen-myeloma fusion. The fusion and subsequent cloning were performed according to established methods ${ }^{38}$ with the aid of the AECOM hybridoma facility. Two subclones were found to have strong phospho-Y1325 reactivity with no reactivity to bovine serum albumin, unphosphorylated $\mathrm{Y} 1325$, or to phosphorylated Y1423 and Y1184, demonstrating the site- and phospho-specificity of our antibodies. Both subclones, termed clones 11.1 and 11.2 , were isotype $\lg _{2 A}$, as determined using the Roche mouse isotyping kit.

\section{Immunofluorescent Labeling and Analysis of Cultured Neurons}

For immunofluorescence studies, human primary neurons were plated on glass cover slips in 24-well dishes in Neurobasal media supplemented with $\mathrm{N}_{2}, 1 \%$ fetal bovine serum and Pen/Strep, and were cultured for up to 28 days. After treatment, cover slips were washed with PBS, fixed in permeabilized ice-cold $70 \%$ ethanol and stored 
at $-20^{\circ} \mathrm{C}$ until immunostaining. Cover slips were rehydrated with 1X PBS before blocking with blocking solution consisting of $5 \mathrm{mmol} / \mathrm{L}$ EDTA, $1 \%$ horse serum, $1 \%$ Ig free bovine serum albumin, and $1 \%$ fish gelatin in PBS. Cover slips were incubated overnight in primary antibody (antiMAP2, 1:200) diluted in blocking solution or hybridoma supernatant from pY1325 clone 11.1 (used neat), washed with PBS, and then incubated in secondary antibody, anti-mouse Alexa Fluor 488 (1:200), and anti-rabbit Alexa Fluor 594 (1:200) for 1 hour at room temperature. After washing with PBS, cover slips were mounted onto slides using ProLong Gold antifade reagent with DAPI (to identify nuclei) and imaged by confocal microscopy. The number of pixels per cell for pY1325 and MAP-2 staining was determined using ImageJ software by setting the minimum signal threshold and measuring the number of positive pixels in a given frame. Data are reported as pY1325 fluorescence in ratio to MAP-2 to control for the number/size of the neurons per frame.

\section{Immunofluorescent Labeling and Analysis of Human Tissue Samples}

Postmortem human tissue sections from cortex and hippocampus of uninfected controls, HIV-infected individuals without encephalitis, and individuals with HIV encephalitis (HIVE) ( $n=4$ cases for each condition, two slides for each anatomical location per individual case) were analyzed by double immunohistochemical staining for MAP-2, a neuronal marker, and NR2A-pY1325 using hybridoma supernatant. Sections of human formalin-fixed, paraffin-embedded tissue $10 \mu \mathrm{m}$ thick were obtained from individuals with similar postmortem intervals. The ages of the individuals were similar for uninfected and HIVE conditions and gender was equally distributed. In the case of HIVE, low CD4 counts were reported before the time of death, and all HIVE cases were cognitively compromised.

The tissue sections were deparaffinized and then incubated for 12 hours on a UV light box, and subsequently incubated 12 hours on a light box in the visible spectrum to reduce background autofluorescence. After antigen retrieval, the sections were incubated in blocking solution ( $5 \mathrm{mmol} / \mathrm{L}$ EDTA, $1 \%$ fish gelatin, $1 \%$ essentially Ig-free bovine serum albumin, and 2\% horse serum) for 1 hour at room temperature and then incubated with anti-MAP2 antibody (1:300) and anti-pY1325 hybridoma supernatant $(1: 20)$ overnight at $4^{\circ} \mathrm{C}$. The sections were then washed with PBS, incubated with fluorescein isothiocyanate-conjugated anti-mouse IgG (Sigma Chemical) or Alexa Fluor 594-conjugated anti-rabbit IgG for 1 hour at room temperature, followed by serial washes in PBS for 1 hour. Samples were then mounted using Prolong Gold antifade reagent (Molecular Probes) and examined by confocal microscopy. Specificity was confirmed by replacing the primary antibody with the appropriate isotype-matched control reagent, anti-IgG2A or the IgG fraction of normal rabbit serum (Santa Cruz Biotechnology). Quantification of the NR2A Y1325 and MAP-2 staining in uninfected, HIV-infected tissue without encephali- tis, and HIVE tissue sections was performed using NIS Elements Advanced Research software (Nikon, Japan) to determine the total and the mean intensity of fluorescence in each channel. NR2A Y1325 was in the green channel and MAP-2 staining was in the red channel. The background obtained with the respective irrelevant antibodies was subtracted before the analyses.

\section{Results}

\section{HIV-tat Treatment of Human Neurons Induces Tyrosine Phosphorylation of the NMDAR Subunit $2 A$}

We previously demonstrated that tat treatment of human cortical neurons induced the formation of a macromolecular complex consisting of LRP, PSD-95, NMDAR, and nNOS at the plasma membrane, and that this complex participated in tat induced neuronal apoptosis, mainly through NMDAR activation and NO production. ${ }^{9}$ The NMDAR found in the complex were composed of NR1 and NR2A subunits. There was little to no NR2B expressed in our culture system (data not shown). As tat induced apoptosis is dependent on NMDAR activation, ${ }^{8,9}$ we examined NMDAR phosphorylation after tat treatment of neuronal cultures as a mechanism of dysregulation of NMDAR activity that can lead to cell death.

Human primary neuronal cultures were treated with tat for $5,10,15,30$, and 60 minutes (30 and 60 minutes not shown, no significance compared with control) and tyrosine phosphorylation of the NR2A subunit was examined. Immunoprecipitation analyses indicated that tat treatment induced robust tyrosine phosphorylation of the human NR2A subunit over control levels. Maximal tyrosine phosphorylation was detected between 5 and 15 minutes after tat treatment (Figure $1 \mathrm{~A}, P<0.01$, IP: NR2A, WB: P-Tyr). The maximal time point of phosphorylation was somewhat variable, due to the variability inherent in primary cell cultures (Figure 1A, IP: NR2A, WB: P-Tyr, two examples shown). Changes in NR2A phosphorylation were not due to changes in the overall amount of NR2A in the lysates (data not shown), nor to the amount of total NR2A immunoprecipitated (Figure 1A, IP: NR2A, WB: NR2A, loading control). Densitometric analyses of the maximal phosphorylation time point of five independent experiments indicated that tat treatment significantly increased tyrosine phosphorylation of human NR2A as compared with its basal phosphorylation (Figure $1 \mathrm{~B}, n=5,{ }^{\star *} P<0.01$ ).

\section{src Associates with the NMDAR after tat Treatment}

To identify the kinases involved in tat induced tyrosine phosphorylation of the NR2A in human neurons, we analyzed kinases that have been found to regulate NMDAR activity. These kinases include src, pyk2, and fyn. 13,19,39,40 Tat treated cultures of human neurons (5-15 minutes) were analyzed by co-immunoprecipitation for NR2A and 
A

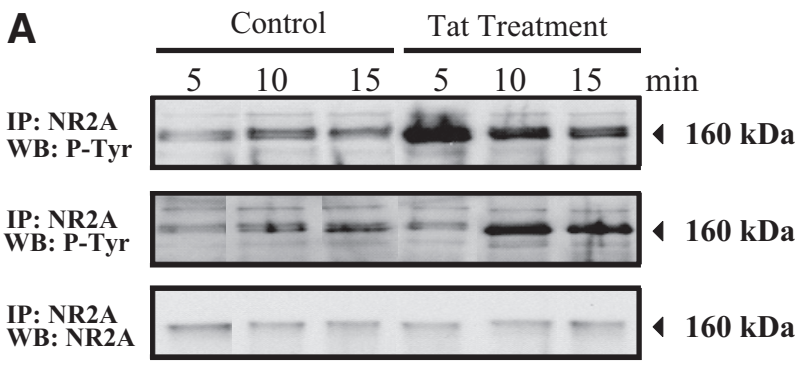

B

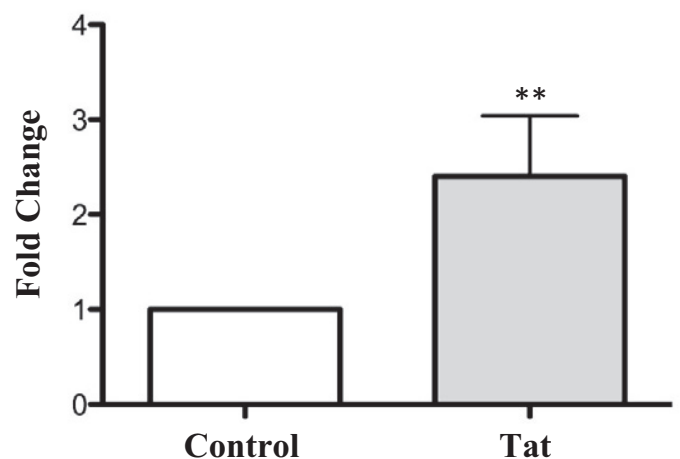

Figure 1. The human NR2A subunit is tyrosine phosphorylated after tat treatment. A: Western blots from two separate, representative experiments demonstrating tat-induced tyrosine phosphorylation of NR2A (IP:NR2A WB P-Tyr). Human neuronal cultures were treated with HIV-tat protein (10 or 100 $\mathrm{ng} / \mathrm{ml}$ ) for 5-60 minutes; 5-15 minutes shown. After cell lysis, NR2A was immunoprecipitated and electrophoresed. Membranes were probed with a pan phosphotyrosine antibody. The time of maximal phosphorylation varied among experiments, but always occurred between 5 and 15 minutes in patterns similar to those represented. A representative control (IP:NR2A WB:NR2A) demonstrates that changes in tyrosine phosphorylation were not due to changes in the total amount of NR2A immunoprecipitated. B: Densitometric analyses of the maximal time point for each individual experiment, normalized to the amount of NR2A immunoprecipitated, indicated a significant increase in the tyrosine phosphorylation of NR2A after tat treatment. (*** $P<0.01, n=5,10$, or $100 \mathrm{ng} / \mathrm{ml}$ of tat).

subsequent Western blotting for total and active src, pyk2, and fyn.

Significant interactions between both active, pY416Src, and total src and NR2A occurred at 5 minutes after tat treatment of neuronal cultures (Figure 2, A-C, $n=3$, $\left.{ }^{\star} P<0.05\right)$. This association corresponds to the time frame of enhanced tyrosine phosphorylation of the NR2A subunit after tat treatment (Figure 1). Changes in src association were not due to changes in the total amount of NR2A immunoprecipitated (Figure 2A, loading control) or to nonspecific immunoprecipitation of NR2A or src, as demonstrated by using an irrelevant lgG1 reagent (Figure 2A, IgG1 IPs). Densitometric analyses of those coimmunoprecipitations indicated that src (active and total)-NR2A interaction was increased after tat treatment over basal interaction (Figure 2, B and $\mathrm{C}, n=3,{ }^{\star} P<$ 0.05). As src-NR2A interaction always coincided with phosphorylation of NR2A, we proposed that src was responsible for tat induced phosphorylation of NR2A in human neurons.

In addition, pyk2, another tyrosine kinase shown to be associated with the NMDAR in other experimental models, ${ }^{39,41,42}$ interacted with NR2A in basal conditions, and this interaction was increased by tat treatment (data not shown). However, pyk2-NR2A association occurred 15 to
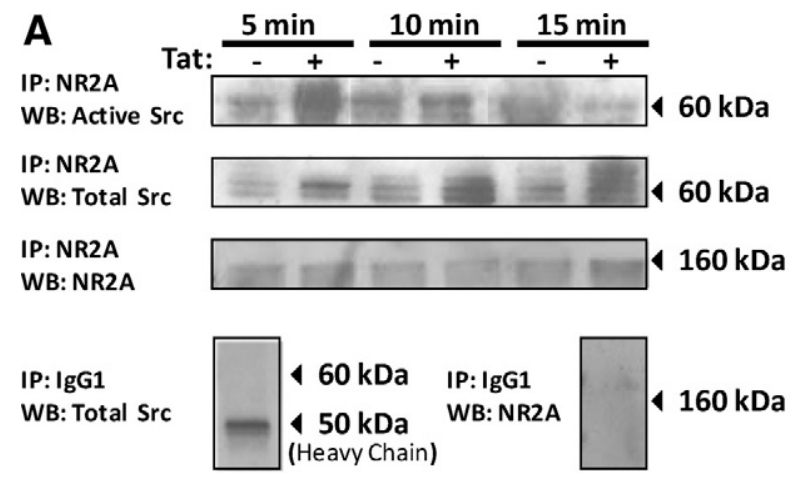

B

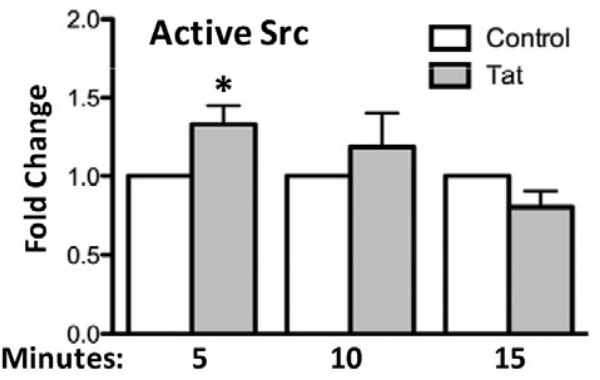

C

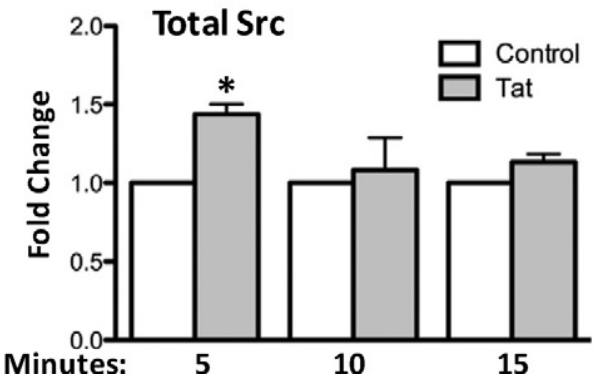

Figure 2. Src is associated with the NMDAR after tat treatment. A: Western blots demonstrating association of active (IP:NR2A WB:Active src) and total src (IP:NR2A WB:Total src) association with NR2A by co-immunoprecipitation and representative control Western blot showing approximately equal amounts of total NR2A immunoprecipitated (IP:NR2A WB:NR2A). Neuronal cultures were treated with tat $(10 \mathrm{ng} / \mathrm{ml})$ for 5,10 , and 15 minutes. NR2A was immunoprecipitated from the lysates, and membranes were probed for active src, total src, and NR2A. Changes in association were not due to nonspecific immunoprecipitation of src or NR2A proteins (IP: IgG1 WB: NR2A and WB src). $\mathbf{B}$ and $\mathbf{C}$ : Densitometric analyses of active $\operatorname{src}\left({ }^{*} P<0.05, n=3\right)(\mathbf{B})$ and of total $\operatorname{src}\left({ }^{*} P<0.05, n=3\right)(\mathbf{C})$ indicated that maximal association with the NMDAR occurred five minutes after tat treatment.

30 minutes (data not shown) after tat-induced tyrosine phosphorylation of the NR2A. The kinase fyn did not associate with NR2A at baseline or after tat treatment (data not shown).

\section{Tat-Induced Tyrosine Phosphorylation of NR2A in Human Neuronal Cultures Is Dependent on src Activity}

As active and total src had increased associations with the NR2A after tat treatment at time points corresponding to tyrosine phosphorylation of NR2A, we hypothesized that src was participating in its phosphorylation. A selective dual-site competitive inhibitor of src, Src kinase inhibitor 1 (Srcl), ${ }^{43}$ was used in human neuronal cultures in the presence or absence of tat treatment, and tyrosine 
A

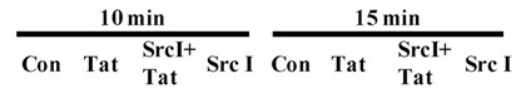

IP:NR2A

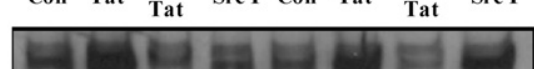

$4160 \mathrm{kDa}$

IP:NR2A WB: NR2A

B

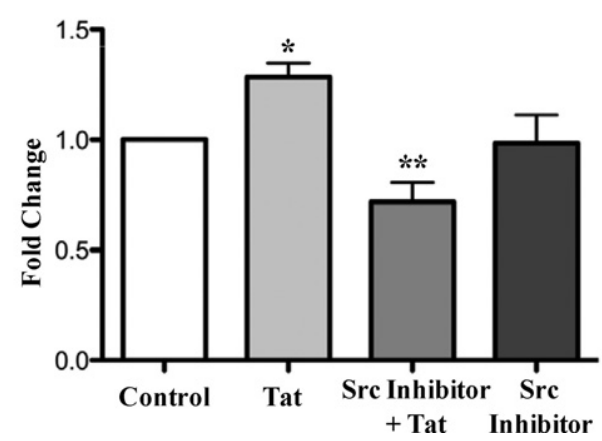

Figure 3. Src activity is necessary for tat-induced tyrosine phosphorylation of NR2A. A: Western blots demonstrating reduced phosphorylation after tat treatment when src activity is inhibited. Neurons were pretreated with 5 $\mu \mathrm{mol} / \mathrm{L}$ src inhibitor, SrcI, or vehicle for 10 minutes before tat treatment (10 or $100 \mathrm{ng} / \mathrm{ml}$ ). NR2A was immunoprecipitated, and Western blotting was performed for phosphotyrosine (IP:NR2A WB:P-Tyr) or NR2A (IP:NR2A WB: NR2A). Tat induced tyrosine phosphorylation of NR2A, which was blocked by pretreatment with $\mathrm{src}$ inhibitor. B: Densitometric analyses indicated that tat induced tyrosine phosphorylation of NR2A ( ${ }^{*} P<0.05$, Control versus Tat), and pretreatment with the src inhibitor prevented tat induced phosphorylation ( ${ }^{* *} P<0.01$, Tat versus SrcI + Tat). Although the src inhibitor sometimes induced minimal phosphorylation, it was not significant as compared with control levels (Control versus Src Inhibitor, not significant) $(n=3)$.

phosphorylation of NR2A was examined by immunoprecipitation. Cells were pretreated with $5 \mu \mathrm{mol} / \mathrm{L} \mathrm{Srcl}$ or vehicle as control for 10 minutes to block src activity before treating with tat for 5,10 , or 15 minutes, the time points at which NR2A tyrosine phosphorylation was found to be maximal (Figure 1). Tat induced tyrosine phosphorylation of NR2A significantly over basal levels (Figure 3, A and $\mathrm{B}, n=3,{ }^{*} P<0.05$, tat), and pretreatment with SrCl abolished this increase induced by tat treatment (Figure $3, A$ and $B, n=3, \# \# P<0.01$, Src Inhibitor + tat). Although src inhibitor alone caused an occasional induction of tyrosine phosphorylation, it was not significantly different from control conditions (Figure $3, \mathrm{~A}$ and $\mathrm{B}, n=$ 3, not significant, Src Inhibitor). Changes in tyrosine phosphorylation induced by tat treatment were not due to changes in the amounts of total NR2A in the immunoprecipitations, as Western blotting showed little difference in total NR2A (Figure 3A, IP:NR2A; WB: P-Tyr). These experiments demonstrated that src activity is necessary for tat-induced tyrosine phosphorylation of NR2A.

\section{NR2A Tyrosines 1184, 1325, and 1423 Are Phosphorylated by src in Vitro}

To identify the tyrosine residues in the cytoplasmic tail of the human NR2A that could be phosphorylated by src, in vitro kinase assays were performed. Using the human amino acid sequence of the NR2A, synthetic peptides were generated containing tyrosines that were likely to be phosphorylated using phosphorylation prediction soft-

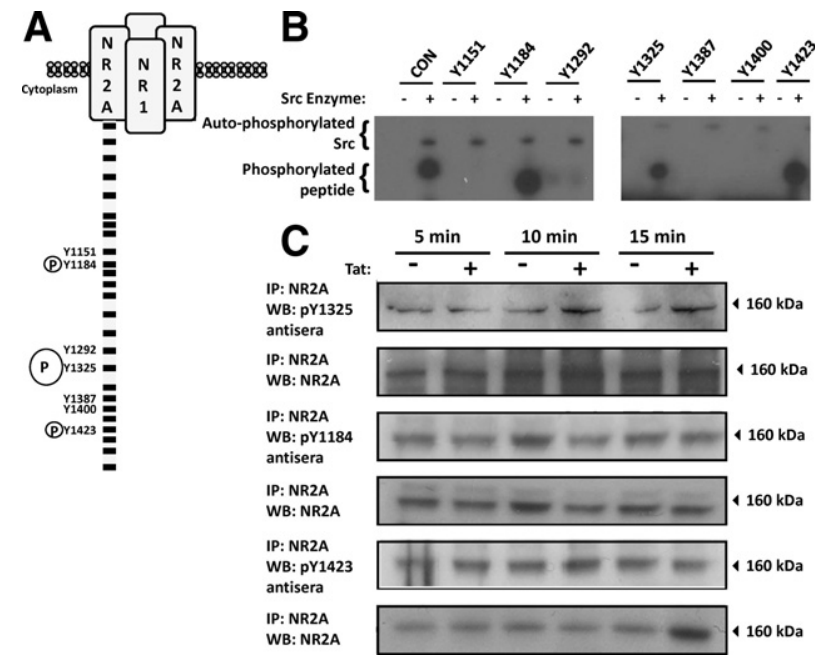

Figure 4. Identification of phosphorylation sites on human NR2A. A: Schematic depicting the tyrosine residues in the cytoplasmic tail of NR2A that were predicted to have a high likelihood of being phosphorylated according to the computer analysis program NetPhos 2.0. The tyrosines marked with a circled P were phosphorylated in our in vitro kinase assay, and Y1325, marked in red, was also found to be phosphorylated after tat treatment in our culture system. B: In vitro kinase assay. Pure recombinant src was preincubated in reaction buffer with ATP for 5 minutes, allowing for src to transautophosphorylate and become maximally active before adding target peptides. CON, src target consensus sequence, our positive control, and Y\# indicates the tyrosine residue of the NR2A cytoplasmic tail. Three of seven residues tested were capable of being phosphorylated in vitro, Y1184, Y1325, and Y1423 ( $n=3)$. C: Y1325 is phosphorylated in our neuronal culture system after tat treatment. Immune sera were generated against the phosphorylated forms of Y1184, Y1325 and Y1423, preabsorbed with the corresponding unphosphorylated peptide, and used for Western blotting after immunoprecipitation for NR2A. Western blotting indicated that Y1325 was a likely target of tat induced phosphorylation. Phosphorylation of Y1184 and Y1423 was present basally but did not change in response to tat treatment $(100 \mathrm{ng} / \mathrm{ml})$.

ware, NetPhos $2.0,^{36}$ or that were sequences that had been examined previously in other systems as potential src sites ${ }^{44}$ in NR2A (Figure 4A, diagram). A total of seven peptides were synthesized as well as the src target consensus sequence as a positive control: src target consensus (EEVYQQWSQ from ${ }^{37}$ ), Y1151 (FPDPYQDPS), Y1184 (NNDQYKLYS), Y1292 (RQHSYDNIV), Y1325 (EGNFYGSLF), Y1387 (PSPPYKHSL), Y1400 (VNDSYLRSS), and Y1423 (HNDVYISEH). In vitro kinase assays showed that tyrosines $Y 1184, Y 1325$, and $Y 1423$ corresponding to the human sequence of NR2A were phosphorylated by src (Figure $4 \mathrm{~B}, n=3$ ) and that there was autophosphorylation of $\operatorname{src}$ (Figure $4 \mathrm{~B}, n=3$ ). Interestingly, of the three tyrosines homologous to the mouse NR2A subunit that had been previously identified in the literature as potential src targets, Y1292, Y1325, and Y1387, ${ }^{44}$ only one, Y1325, was phosphorylated by src in our in vitro kinase assay (Figure 4B). Tyrosines 1151, 1292, 1387, and 1400 were not phosphorylated by src in vitro (Figure 4B, $n=3$ ).

\section{Generation of Phospho Site-Specific Antibodies and Identification of Y1325 as a tat-Sensitive Phosphorylation Site on the Human NR2A}

Immune sera were generated in mice to the phosphorylated forms of those peptides found to be phosphorylated 
in our in vitro kinase assay, specifically Y1184, Y1325, and $\mathrm{Y} 1423$, to determine whether the sites phosphorylated in vitro were phosphorylated in human primary neuronal cultures after tat treatment. To test for phosphorylation of these three tyrosines, cell cultures were treated with tat for 5, 10, and 15 minutes, lysates were prepared, and immunoprecipitation for NR2A and Western blotting using the immune sera for each phosphorylated peptide was performed. We detected basal tyrosine phosphorylation of the three residues analyzed, Y1184, Y1325 and $Y$ 1423. In addition, phosphorylation of $Y 1325$ was significantly increased after tat treatment over control conditions (Figure 4C). Changes in phosphorylation of Y1325 were not due to changes in the total amount of NR2A immunoprecipitated, as determined by Western blot analysis (Figure 4C).

To examine this tat sensitive phosphorylation site of the NR2A, we generated phospho site-specific monoclonal antibodies against pY1325 according to standard procedures $^{38}$ (AECOM hybridoma facility). Reactivity of the hybridoma supernatants to both the phosphorylated and unphosphorylated Y1325 peptides was determined to confirm phosphorylation specificity. Reactivity to the phosphorylated $Y 1325$ peptide titered to $>1: 20,000$ dilution. Reactivity to the unphosphorylated form was not above background absorbance (Figure 5A). No nonspecific reactivity was detected to pY1184 (data not shown) and pY1423, demonstrating site specificity, nor to bovine serum albumin (Figure 5A), which was in the screening enzyme-linked immunosorbent assay diluent.

The generated phospho site-specific monoclonal antibody (pY1325 mAb) was then used for Western blotting after immunoprecipitation for NR2A from lysates generated from untreated and tat treated $(5,10$, and 15 minutes) primary human neurons. Consistent with the results obtained using immune sera, Western blotting with pY1325 mAb showed some baseline phosphorylation in control conditions (Figure 5B). Tat treatment significantly increased phosphorylation of residue $\mathrm{Y} 1325$ of NR2A (Figure 5, B and $\mathrm{C},{ }^{* \star} P<0.01$ ), corresponding to the time in which we detected tyrosine phosphorylation with the pan-phosphotyrosine antibody and with mouse immune sera (Figures 1 and 4). Changes in pY1325 were not due to changes in the total amount of NR2A immunoprecipitated (Figure 5B, IP:NR2A; WB: NR2A).

To determine whether phosphorylation of $\mathrm{Y} 1325$ was dependent on src activity, we performed confocal analyses of human neuronal cultures that were treated with tat, srcl + tat, srcl alone and vehicle (Figure 6) and were stained using the pY1325 mAb. Cultures were treated with $\mathrm{Srcl}(5 \mu \mathrm{mol} / \mathrm{L})$ or vehicle for 10 minutes before treating with tat for 5, 10, and 15 minutes. Immunostaining with pY1325 mAb hybridoma supernatant and MAP-2 antibody, a neuronal marker, or the appropriate antibody controls was performed. Tat treatment of neuronal cultures for 5 minutes significantly increased the staining for pY1325 along the cell body and neuronal processes (green, Figure 6, D-F, M, ${ }^{\star \star} P<0.01$ ), as compared with cells that received vehicle treatment (Figure 6, A-C). Pretreatment with $\mathrm{srcl}$ blocked the tat-induced increase in phosphorylation of Y1325 (Figure 6, G-I) as compared
A
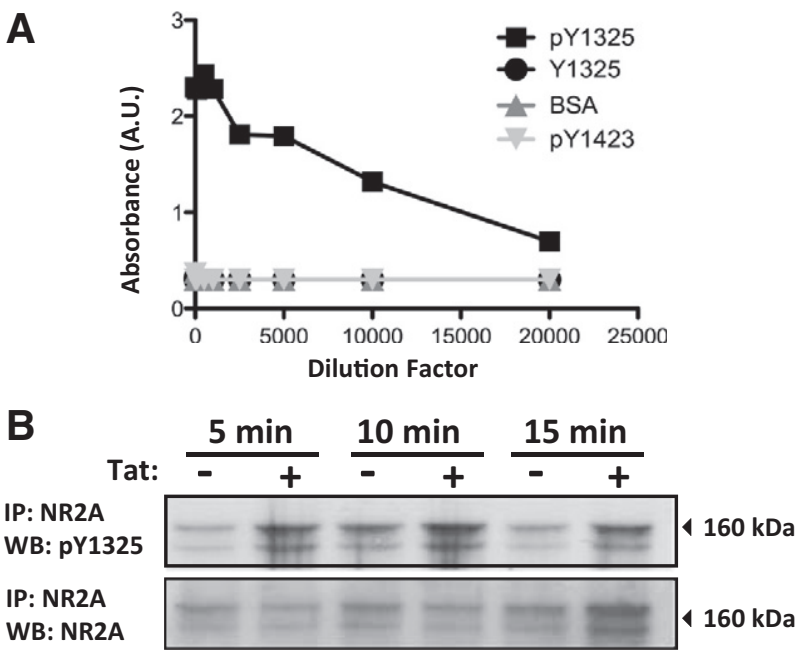

C

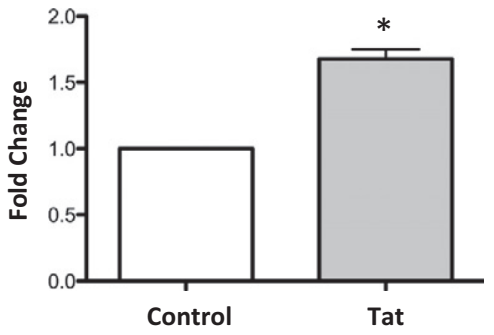

Figure 5. Monoclonal antibodies to phosphorylated Y1325 were specific and NR2A was phosphorylated on Y1325 after tat treatment. A: Monoclonal antibodies from clone 11.1 were specific for phosphorylated Y1325. Hybridoma supernatant collected from clone 11.1 was diluted up to $1: 20,000$ in $1 \%$ bovine serum albumin in PBS, and enzyme-linked immunosorbent assays were performed. The supernatant titered to 1:20,000 against phosphorylated Y1325 (pY1325, black square), but did not recognize the unphosphorylated form of the peptide (Y1325, black circle), other phosphorylated peptides (pY1423, gray inverted triangle), or bovine serum albumin (gray triangle), used in the blocking solution. B: Y1325 is phosphorylated after tat treatment. Neuronal cultures were treated with tat $(100 \mathrm{ng} / \mathrm{ml})$ for 5-30 minutes, and NR2A was immunoprecipitated from the lysates. Western blotting was performed with the hybridoma supernatant, diluted 1:1000. Phosphorylation occurred between 5 and 15 minutes after tat treatment (IP:NR2A WB: pY1325). C: Densitometric analyses demonstrated that NR2A was phosphorylated at Y1325 after tat treatment $\left({ }^{*} P<0.01, n=4,100\right.$ or $300 \mathrm{ng} / \mathrm{ml}$ tat $)$

with tat treatment alone (Figure 6M, \#\#P<0.01,). Src kinase inhibitor alone had no effect on the phosphorylation of Y1325 compared with control cells (Figure 6, J-M). No background or nonspecific staining was detected using irrelevant isotype matched antibodies or irrelevant immune hybridoma supernatant (data not shown). Quantification of the pixels positive for NR2A-pY1325 and MAP-2 was performed and the data were expressed as the ratio of NR2A-pY1325/MAP-2, to adjust for numbers of neurons in each frame (Figure 6M). For all conditions, only neurons with some pY1325 staining were examined.

\section{Increased Phosphorylation of NR2A Y1325 in Brain Tissue Sections Obtained from Individuals with HIV Encephalitis (HIVE)}

As tat induced phosphorylation of $\mathrm{Y} 1325$ in human neuronal cells, we examined phosphorylation of $Y 1325$ of the NMDAR in brain tissue sections obtained from uninfected individuals (Figure 7, Control), HIV-infected individuals 

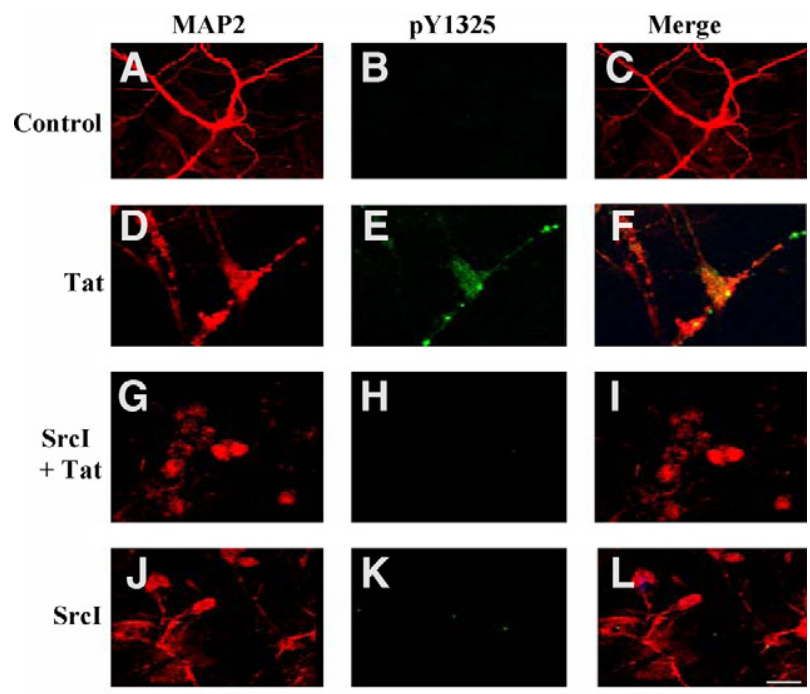

M

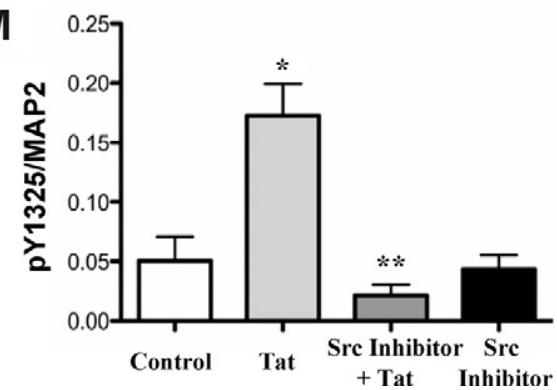

Figure 6. Immunofluorescent staining indicates that tat treatment induces increased phosphorylation of Y1325 and that this is blocked by treatment with src inhibitor. A-L: Neuronal cultures were pretreated with SrcI or vehicle for ten minutes before being treated with tat $(300 \mathrm{ng} / \mathrm{ml})$ or vehicle for 5,10 , and 15 minutes. The 5-minute treatment is shown. Cover slips were subsequently fixed and stained for MAP2 (red), a neuronal marker, and pY1325 (green). Overlay of pY1325 and MAP2 is shown in the right-hand column (merge). Tat treatment (D-F) induced an increase in phosphorylation over controls $(\mathbf{A}-\mathbf{C})$. Pretreatment for ten minutes with src inhibitor prevented tat induced phosphorylation $(\mathbf{G}-\mathbf{I}, \mathbf{M})$. SrcI treatment alone did not affect phosphorylation at the time point examined $(\mathbf{J}-\mathbf{L})$. M: The number of pixels per frame of each color was counted, and the ratio of green pixels to red pixels was calculated. Only those images with at least one spot of pY1325 staining were counted. Tat induced enhanced phosphorylation $\left({ }^{*} P<0.05\right)$. Src inhibitor blocked tat induced phosphorylation ( ${ }^{* * *} P<0.01$, compared with tat treatment). There was no difference between control and SrcI + tat treated cultures ( $n=3$, with at least three pictures for each cover slip and two cover slips per experiment).

without encephalitis (HIV) and from individuals with HIV encephalitis (HIVE) using immunofluorescent labeling and confocal microscopy. MAP-2 immunostaining was used to identify neurons in the tissue sections and our pY1325 mAb was used to show phosphorylation at this site.

Brain tissue sections obtained from uninfected individuals and HIV-infected individuals without encephalitis showed very low levels of pY1325 mAb immunolabeling in both the cortex (green, Figure 7, B and E, respectively) and hippocampus (data not shown) in neuronal bodies identified by staining with MAP-2 (red, Figure 7, A and D). Tissue sections obtained from individuals with HIVE had highly increased staining with pY1325 mAb in MAP-2 positive cells, especially on the cell bodies of these neurons in both the cortex (Figure 7, G and $\mathrm{H}$ ) and hippocampus (data not shown) as compared with sections obtained from uninfected (Figure $7, \mathrm{~A}-\mathrm{C}$, Control) and HIV-infected individuals without encephalitis (Figure 7 , D-F, HIV). The increased staining with pY1325 mAb in the HIVE tissue was not due to ischemic insult from the postmortem interval before sample collection, as this was similar in all of the cases analyzed.

Quantification of the total fluorescence or the mean fluorescence intensity of all pictures obtained for NR2A Y1325 and MAP-2 using NIS Elements Advance Research software, indicate that control brain sections have a total intensity of Y1325 staining of 440,890 $\pm 201,017$ arbitrary units (A.U.) with a mean intensity of $1.71 \pm 0.75$ A.U. HIV-infected brains without encephalitis have a total intensity of $Y 1325$ staining of 508,661 $\pm 202,219$ with a mean intensity of $1.738 \pm 0.87$ A.U. and HIVE tissue sections have a total intensity of Y1325 staining of $1,004,191 \pm 424,008$ with a mean fluorescence intensity of $3.59 \pm 1.26$ A.U. These results indicate that control and HIV sections are not significantly different in total or mean intensity of fluorescence $(P=0.373$ and $P=0.934$, respectively). In contrast, control or sections from HIVinfected individuals without encephalitis were significantly different from HIVE sections in total and mean fluorescence (control versus HIVE, $P=2.49 \times 10^{-5}$ and $P=9.12 \times 10^{-6}$, respectively and HIV versus HIVE, $P=$ 0.00012 and $P=1.79 \times 10^{-5}$, respectively). MAP-2 staining quantification of the human tissue sections showed that control brain sections have a total intensity of MAP-2 staining of 4,410,594 $\pm 740,646$ with a mean intensity of $16.54 \pm 2.94$ A.U. Sections from HIV brains without encephalitis have a total intensity of MAP-2 staining of 4,414,144 $\pm 1,004,439$ with a mean intensity of $16.27 \pm 3.87$ A.U. and HIVE tissue sections have a total intensity of MAP-2 staining of 3,104,139 $\pm 1,532,179$ with a mean intensity of $10.86 \pm 5.08$ A.U. These results indicate that control and HIV without encephalitis brain sections are not statistically significantly different in total and mean intensity of fluorescence $(P=0.9913$ and $P=$ 0.828 , respectively). In contrast, control or HIV without encephalitis sections were significantly different from HIVE sections in total and mean fluorescence (control versus HIVE, $P=0.00313$ and $P=0.000344$, respectively, and HIV without encephalitis versus HIVE, $P=$ 0.00529 and $P=0.00132$, respectively).

Thus, the total amount of Y1325 was higher in the HIVE sections as compared with control and HIV tissue sections without encephalitis. MAP-2 staining in HIVE was lower than that for the control brains or HIV-infected brains without encephalitis, possibly indicating loss and/or damage of neurons in HIVE as described previously. ${ }^{45}$ Isotype matched control antibodies were negative and were used as background controls for the quantification of fluorescence (data not shown). All cases from the same condition had similar staining patterns as the representative pictures shown in Figure 7.

\section{Discussion}

Our results demonstrate that HIV-tat increases tyrosine phosphorylation of Y1325 on the human NMDAR subunit 

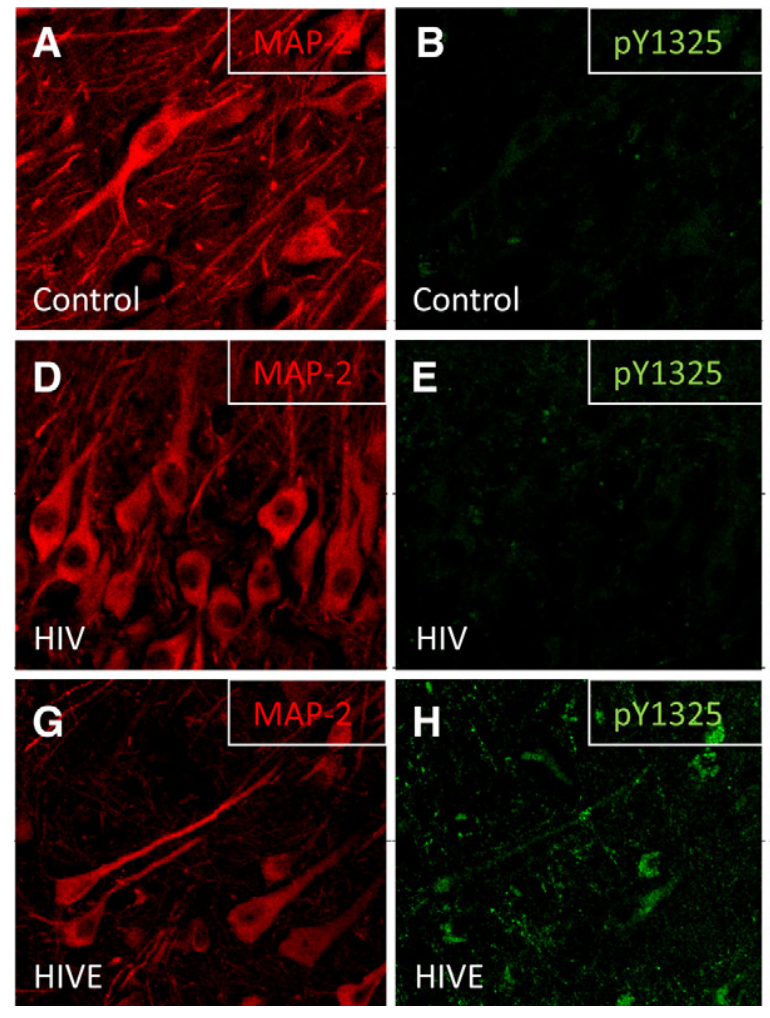
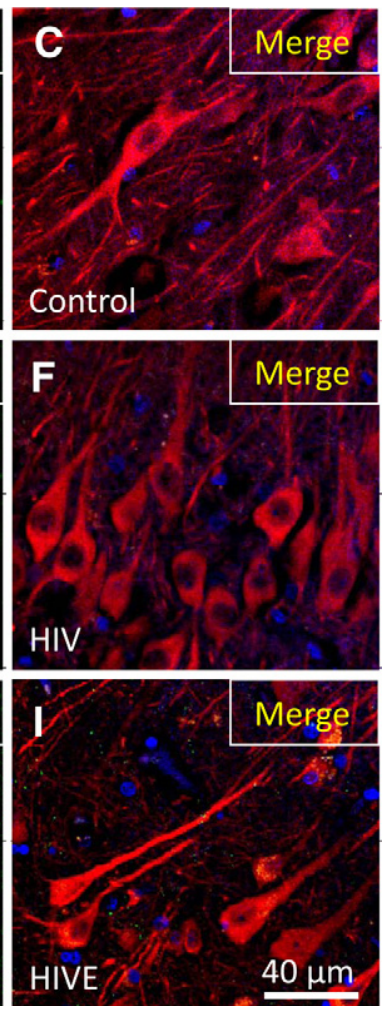

Figure 7. Phosphorylation of Y1325 of NR2A is increased in HIV encephalitic tissue. Tissue sections from HIV encephalitic brains (HIVE, $n=$ 4), HIV-infected without encephalitis (HIV) or from control brains (Control) with a similar interval to autopsy and fixation $(n=4)$ were immunostained for MAP-2 (red, $\mathbf{A}, \mathbf{D}$, and $\mathbf{G}$ ), a neuronal marker and phosphorylated Y1325, labeled pY1325 (green, B, E, and $\mathbf{H}$ ). Micrographs labeled $\mathbf{C}, \mathbf{F}$, and $\mathbf{I}$ are the merged images. $\mathbf{A}-\mathbf{C}$ : Control tissue demonstrated robust MAP-2 staining and minimal staining for phosphorylated Y1325. D-F: HIV tissue sections without encephalitis (HIV) show a similar minimal staining pattern to control conditions. The merged images show that all Y1325 staining colocalizes with MAP-2 and thus is found only in the neurons $(\mathbf{C}$ and $\mathbf{F})$. HIV encephalitic tissue had greatly increased staining for pY1325 relative to control levels. There was also diminished MAP-2 staining relative to controls cells, perhaps indicative of neuronal damage, as there were also fewer neurons. The MAP-2 staining is so intense for all panels, such that in the merged images it is difficult to see the colocalization of green and red (orange). However, the reactivity with pY1325 is very apparent in $\mathbf{H}$, as compared with $\mathbf{B}$ (Control) and $\mathbf{E}$ (HIV). The quantification of the fluorescence intensity for Y1325 and MAP-2 staining are described in Results.
$2 \mathrm{~A}$ in our primary human neuronal cultures by recruitment and activation of src. Additionally, we found increased phosphorylation of Y1325 in human neuronal cultures and in HIV encephalitic brain sections as compared with uninfected control and HIV-infected without encephalitis brain sections. Thus, our results identify a specific tyrosine residue, Y1325, in the human NR2A whose phosphorylation is dependent on src activity, and increased in response to tat in cultured neurons. Additionally, phosphorylation of the NR2A Y1325 residue was significantly increased in neurons in human brain tissue sections in the context of HIVE as compared with sections obtained from uninfected and HIV-infected individuals without encephalitis.

Aberrant activity of the NMDAR is involved in the tat induced apoptotic process of human neuronal cultures, as blocking the NMDA receptor with MK801 or AP-5 inhibited tat induced apoptosis. ${ }^{8,9}$ Additionally, our previous results demonstrated that tat treatment alters protein-protein interactions, channel activity and aggregation of NMDAR channels on the surface of human neurons. ${ }^{8,9}$ Phosphorylation of NMDAR subunits has been shown to affect these processes. ${ }^{13-17}$ The NMDAR can be phosphorylated on serine/threonine residues by protein kinase $A$, protein kinase C, CaMKII and casein kinase II, ${ }^{46}$ as well as on tyrosine residues by fyn, src, and other kinases. ${ }^{46}$ Aberrant phosphorylation has been observed in several central nervous system pathologies. ${ }^{47-49}$ However, most studies of these diseases were performed in rat and mouse model systems, in which NMDAR differs from the human in subunit composition and amino acid sequence. We are now examining HIV tat induced tyrosine phosphorylation of the human NMDAR subunit 2A in human neurons as a mechanism that participates in the pathogenesis of neuroAIDS.

Our results demonstrate that tat induces tyrosine phosphorylation of $\mathrm{Y} 1325$ on the NR2A subunit in human neurons through the recruitment and activation of src and its interaction with the NMDAR. Src family kinases have been shown to regulate NMDAR activity in a variety of ways, including insertion of new channels onto the surface of neurons ${ }^{50}$ and in the potentiation of NMDAR currents. ${ }^{12,19,44,51}$ Further, others have shown using recombinant expression systems that src increased the glutamate induced current of NR2A containing receptors, but not those receptors containing exclusively NR2B, NR2C or NR2D. ${ }^{12}$ We propose that up-regulation of src activity is a mechanism of tat induced activation of NR2A containing NMDAR in our system. The effects of src on tat induced activation are currently being examined.

We found that maximal tyrosine phosphorylation occurred between 5 and 15 minutes after tat treatment. We previously demonstrated the formation of a tat induced membrane complex among LRP, PSD-95, and the NMDAR that participates in tat mediated apoptosis. ${ }^{8,9,12}$ Formation of this complex on the neuronal membrane was maximal at 15 to 30 minutes, ${ }^{9}$ time points after maximal tyrosine phosphorylation. Thus, formation of the complex may also be down-regulating src activity, as PSD-95 can be a negative regulator of src at the NMDAR. ${ }^{52-54}$

In addition to src, fyn ${ }^{20,21}$ and pyk2 $2^{39,55}$ kinases have also been implicated in NMDAR activation. However, we did not find fyn associated with the NMDAR in control or tat-treated human cells (data not shown). In contrast, pyk2 was associated with NR2A (data not shown), but the time course of interaction (15 to 30 minutes) did not 
correlate with the tyrosine phosphorylation of NR2A, suggesting that pyk2 may have a role in the subsequent events of complex formation and nNOS activation ${ }^{9}$ but not in the initial phosphorylation of Y1325 of NR2A after tat treatment.

Previous reports in rat neurons found that tat induced tyrosine phosphorylation of NR2A and NR2B after 40 minutes of treatment through PKC, $\mathrm{IP}_{3}$, and G-proteinmediated pathways. ${ }^{10,56}$ The difference in the kinetics of our data may be due to underlying species differences. We observed interspecies differences in the sensitivities of these cultures to tat induced apoptosis. Rat hippocampal neurons have much lower levels of apoptosis in response to tat treatment. Our data and other published reports indicate 5 to $35 \%$ apoptosis in rat neurons, ${ }^{7,56}$ as compared with the 65 to $90 \%$ apoptosis that we detected in tat treated human neurons. ${ }^{8,9}$ In addition, tat-induced complex formation involves NR2A-containing NMDAR in human neurons, ${ }^{8,9}$ while in rat neurons, we found that the complex is preformed and contains both NR2A and NR2B subunits (data not shown). It has been shown that NMDAR subunits confer different properties that affect receptor function. For example, NR2A receptors have faster deactivation kinetics than NR2B containing receptors, ${ }^{57}$ and they are differentially expressed during development. ${ }^{58}$ It is not yet clear whether the difference in subunit composition between the human and rat, preformation of the complex in rat neurons, and/or alternative signaling contribute to the interspecies differences in apoptosis after tat treatment. It is also important to note that the concentrations of extracellular tat in vivo are unknown. It is possible that the concentrations of tat to be used with cultured neurons may differ considerably depending on species, and tat preparations. ${ }^{59}$

We identified three tyrosines in the cytoplasmic tail of NR2A in human primary neurons that are phosphorylated in vivo under control conditions: Y1184, Y1325, and Y1423. HIV tat treatment of neuronal cultures resulted in increased phosphorylation of $Y 1325$ of NR2A, but not of $\mathrm{Y} 1184$ or $\mathrm{Y} 1423$, indicating that the phosphorylation of this particular tyrosine residue is tat sensitive. A previous study had identified 3 sites on NR2A that were phosphorylated when recombinant mouse NMDAR subunits were coexpressed with V-src in HEK293 cells: Y1292, Y1325, and $Y 1387 .{ }^{44}$ However, of these three residues, only Y1325 was phosphorylated by src in our in vitro kinase assay, and thus we did not examine Y1292 or Y1387 phosphorylation in our culture system.

We found that $Y 1325$ is phosphorylated after tat treatment both by Western blot analyses and by immunostaining of tat treated neuronal cultures using a phosphospecific monoclonal antibody that we generated to pY1325. Immunostaining was found after tat treatment of cultured neurons in a similar distribution in the cell body and processes to LRP and NMDAR complexes described previously. ${ }^{9}$ The phosphorylation of NR2A on Y1325 was src dependent. Blocking src activity by pretreating with a src inhibitor completely inhibited tat induced phosphorylation of NR2A.

To demonstrate that the findings in our cultured human neurons could be detected in brain tissue sections ob- tained from HIV-infected individuals with encephalitis, we examined and quantified the reactivity of our phospho site-specific antibody to pY1325 with human neurons in cortical and hippocampal tissue sections from control and HIV-infected individuals. Basal phosphorylation of Y1325 was low in control tissue sections. Significantly increased staining for pY1325 was observed in the HIVE tissue examined as compared with staining of control tissue or HIV-infected tissue without encephalitis, demonstrating a potential role of this phosphorylation in the pathogenesis of neuroAIDS. Phosphorylation of this site in the context of other neuroinflammatory conditions cannot be ruled out. However, the individuals from whom these sections were obtained were well characterized with regard to any existing comorbidities. Thus, in these particular sections, the phosphorylation was solely in the context of HIV encephalitis.

Compelling in vitro data suggest the involvement of the NMDAR in HIV pathogenesis ${ }^{3}$ and other neurodegenerative diseases. ${ }^{60}$ Recent clinical trials have attempted to target this receptor for therapeutic purposes. Memantine, a noncompetitive inhibitor of the NMDAR used to treat other neurodegenerative diseases, is currently being investigated for the treatment of HIV neurocognitive disorders. ${ }^{61,62}$

In this report, we demonstrate that tat increases tyrosine phosphorylation of the human NR2A subunit of the NMDAR in cultured human neurons, that src associates with the NMDAR after tat treatment, and that NR2A phosphorylation is dependent on the activity of src. We also identify $Y 1325, \mathrm{Y} 1184$, and $\mathrm{Y} 1423$ of the NR2A subunit as phosphorylation targets of src in vitro, and $\mathrm{Y} 1325$ as a tat-sensitive, src-dependent phosphorylation site in vivo in human neurons. Additionally, phosphorylation of Y1325 was enhanced in human tissue sections obtained from individuals with HIVE. These findings indicate one of the early mechanisms by which tat may alter NMDAR activation and initiate apoptosis in neurons, contributing to the pathogenesis of neurocognitive impairment observed in HIV-infected individuals. Understanding this disease process is important for the development of therapies to prevent or treat the neurocognitive manifestations seen in HAND (HIV Associated Neurocognitive Disorders).

\section{Acknowledgments}

We thank the Human Fetal Tissue Repository, the Analytical Imaging Facility, and the Hybridoma Facility, especially Ms. Susan Buhl, and the CFAR Pathology and Immunology Core at the Albert Einstein College of Medicine. We are also grateful to Dr. Peter Davies, Dr. Matthew Scharff, and Dr. Herbert Tanowitz for their help with antibody production.

\section{References}

1. McArthur JC: HIV dementia: an evolving disease. J Neuroimmunol 2004, 157:3-10

2. Sacktor N: The epidemiology of human immunodeficiency virus-as- 
sociated neurological disease in the era of highly active antiretroviral therapy. J Neurovirol 2002, 8:115-121

3. King JE, Eugenin EA, Buckner CM, Berman JW: HIV tat and neurotoxicity. Microb Infect 2006, 8:1347-1357

4. Bell JE: The neuropathology of adult HIV infection. Rev Neurol (Paris) 1998, 154:816-829

5. Takahashi K, Wesselingh SL, Griffin DE, McArthur JC, Johnson RT, Glass JD: Localization of HIV-1 in human brain using polymerase chain reaction/in situ hybridization and immunocytochemistry. Ann Neurol 1996, 39:705-711

6. Torres-Munoz JE, Nunez M, Petito CK: Successful application of hyperbranched multidisplacement genomic amplification to detect HIV-1 sequences in single neurons removed from autopsy brain sections by laser capture microdissection. J Mol Diagn 2008, 10:317-324

7. Magnuson DS, Knudsen BE, Geiger JD, Brownstone RM, Nath A: Human immunodeficiency virus type 1 Tat activates non-N-methyl-Daspartate excitatory amino acid receptors and causes neurotoxicity. Ann Neurol 1995, 37:373-380

8. Eugenin EA, D'Aversa TG, Lopez L, Calderon TM, Berman JW: MCP-1 (CCL2) protects human neurons and astrocytes from NMDA or HIVtat-induced apoptosis. J Neurochem 2003, 85:1299-1311

9. Eugenin EA, King JE, Nath A, Calderon TM, Zukin RS, Bennett MVL, Berman JW: HIV-tat induces formation of an LRP-PSD-95- NMDARnNOS complex that promotes apoptosis in neurons and astrocytes. Proc Natl Acad Sci USA 2007, 104:3438-3443

10. Haughey NJ, Holden CP, Nath A, Geiger JD: Involvement of inositol 1,4,5-trisphosphate-regulated stores of intracellular calcium in calcium dysregulation and neuron cell death caused by HIV-1 protein Tat. J Neurochem 1999, 73:1363-1374

11. Yao H, Peng F, Dhillon N, Callen S, Bokhari S, Stehno-Bittel L, Ahmad SO, Wang JQ, Buch S: Involvement of TRPC channels in CCL2mediated neuroprotection against tat toxicity. J Neurosci 2009, 29:1657-1669

12. Kohr G, Seeburg PH: Subtype-specific regulation of recombinant NMDA receptor-channels by protein tyrosine kinases of the src family. J Physiol 1996, 492:445-452

13. Wang YT, Salter MW: Regulation of NMDA receptors by tyrosine kinases and phosphatases. Nature 1994, 369:233-235

14. Wang YT, Yu XM, Salter MW: $\mathrm{Ca}(2+)$-independent reduction of $\mathrm{N}$ methyl-D-aspartate channel activity by protein tyrosine phosphatase. Proc Natl Acad Sci USA 1996, 93:1721-1725

15. Chung HJ, Huang YH, Lau L-F, Huganir RL: Regulation of the NMDA receptor complex and trafficking by activity-dependent phosphorylation of the NR2B Subunit PDZ Ligand. J Neurosci 2004, 24:1024810259

16. Scott DB, Blanpied TA, Ehlers MD: Coordinated PKA and PKC phosphorylation suppresses RXR-mediated ER retention and regulates the surface delivery of NMDA receptors. Neuropharmacology 2003, 45:755-767

17. Scott DB, Blanpied TA, Swanson GT, Zhang C, Ehlers MD: An NMDA Receptor ER retention signal regulated by phosphorylation and alternative splicing. J Neurosci 2001, 21:3063-3072

18. Nishizawa Y: Glutamate release and neuronal damage in ischemia. Life Sci 2001, 69:369-381

19. Yu X-M, Askalan R, Keil GJ II, Salter MW: NMDA channel regulation by channel-associated protein tyrosine kinase Src. Science 1997, 275:674-678

20. Hou X-Y, Zhang G-Y, Yan J-Z, Chen M, Liu Y: Activation of NMDA receptors and L-type voltage-gated calcium channels mediates enhanced formation of Fyn-PSD95-NR2A complex after transient brain ischemia. Brain Res 2002, 955:123-132

21. Tezuka T, Umemori H, Akiyama T, Nakanishi S, Yamamoto T: PSD-95 promotes Fyn-mediated tyrosine phosphorylation of the N-methyl-Daspartate receptor subunit NR2A. Proc Natl Acad Sci USA 1999, 96:435-440

22. Huang Y-Q, Lu W-Y, Ali DW, Pelkey KA, Pitcher GM, Lu YM, Aoto H, Roder JC, Sasaki T, Salter MW, MacDonald JF: CAK $\beta /$ Pyk2 kinase is a signaling link for induction of long-term potentiation in CA1 hippocampus. Neuron 2001, 29:485-496

23. Ma J, Zhang G, Liu Y, Yan J, ZB H: Lithium suppressed Tyr-402 phosphorylation of proline-rich tyrosine kinase (Pyk2) and interactions of Lyk2 and PSD-95 with NR2A in rat hippocampus following cerebral ischemia. Neurosci Res 2004, 49:357-362
24. Zalewska T, Ziemka-Nalecz M, Domanska-Janik K: Transient forebrain ischemia effects interaction of src, fak and pyk2 with the NR2B subunit of $\mathrm{N}$-methyl-D-aspartate receptor in gerbil hippocampus. Brain Res 2005, 1042:214-223

25. Aksenova MV, Aksenov MY, Adams SM, Mactutus CF, Booze RM: Neuronal survival and resistance to HIV-1 Tat toxicity in the primary culture of rat fetal neurons. Exp Neurol 2009, 215:253-263

26. Bonavia R, Bajetto A, Barbero S, Albini A, Noonan DM, Schettini G: HIV-1 Tat causes apoptotic death and calcium homeostasis alterations in rat neurons. Biochem Biophys Res Commun 2001, 288:301-308

27. Cheng J, Nath A, Knudsen B, Hochman S, Geiger JD, Ma M, Magnuson DS: Neuronal excitatory properties of human immunodeficiency virus type 1 Tat protein. Neuroscience 1998, 82:97-106

28. Conant K, Garzino-Demo A, Nath A, McArthur JC, Halliday W, Power C, Gallo RC, Major EO: Induction of monocyte chemoattractant protein-1 in HIV-1 Tat-stimulated astrocytes and elevation in AIDS dementia. Proc Natl Acad Sci USA 1998, 95:3117-3121

29. D'Aversa TG, Yu KO, Berman JW: Expression of chemokines by human fetal microglia after treatment with the human immunodeficiency virus type 1 protein Tat. J Neurovirol 2004, 10:86-97

30. Johnston JB, Zhang K, Silva C, Shalinsky DR, Conant K, Ni W, Corbett D, Yong VW, Power C: HIV-1 Tat neurotoxicity is prevented by matrix metalloproteinase inhibitors. Ann Neurol 2001, 49:230-241

31. Kruman II, Nath A, Mattson MP: HIV-1 protein Tat induces apoptosis of hippocampal neurons by a mechanism involving caspase activation, calcium overload, and oxidative stress. Exp Neurol 1998, 154:276-288

32. Li W, Huang $Y$, Reid R, Steiner J, Malpica-Llanos T, Darden TA, Shankar SK, Mahadevan A, Satishchandra P, Nath A: NMDA receptor activation by HIV-Tat protein is clade dependent. J Neurosci 2008, 28:12190-12198

33. Nath A, Conant K, Chen P, Scott C, Major EO: Transient exposure to HIV-1 Tat protein results in cytokine production in macrophages and astrocytes: a hit and run phenomenon. J Biol Chem 1999, 274:17098-17102

34. Perez A, Probert AW, Wang KK, Sharmeen L: Evaluation of HIV-1 Tat induced neurotoxicity in rat cortical cell culture. J Neurovirol 2001, $7: 1-10$

35. Woodman SE, Benveniste EN, Nath A, Berman JW: Human immunodeficiency virus type 1 TAT protein induces adhesion molecule expression in astrocytes. J Neurovirol 1999, 5:678-684

36. Blom N, Gammeltoft S, Brunak S: Sequence- and structure-based prediction of eukaryotic protein phosphorylation sits. J Mol Biol 1999, 294:1352-1362

37. Bi R, Rong Y, Bernard A, Khrestchatisky M, Baudry M: Src-mediated tyrosine phosphorylation of NR2 subunits of N-methyl-D-aspartate receptors protects from calpain-mediated truncation of their C-terminal domains. J Biol Chem 2000, 275:26477-26483

38. French D, Kelly T, Buhl S, Scharff MD: Somatic cell genetic analysis of myelomas and hybridomas. Methods Enzymol 1987, 151:50-66

39. Liu Y, Zhang G, Gao C, Hou X: NMDA receptor activation results in tyrosine phosphorylation of NMDA receptor subunit 2A(NR2A) and interaction of Pyk2 and Src with NR2A after transient cerebral ischemia and reperfusion. Brain Res 2001, 909:51-58

40. Chen M, Hou X, Zhang G: Tyrosine kinase and tyrosine phosphatase participate in regulation of interactions of NMDA receptor subunit $2 \mathrm{~A}$ with Src and Fyn mediated by PSD-95 after transient brain ischemia. Neurosci Lett 2003, 339:29-32

41. Liu Y, Hou X-Y, Zhang G-Y, Xu T-L: L-type voltage-gated calcium channel attends regulation of tyrosine phosphorylation of NMDA receptor subunit 2A induced by transient brain ischemia. Brain Res 2003, 972:142-148

42. Cheung HH, Takagi N, Teves L, Logan R, Wallace MC, Gurd JW: Altered association of protein tyrosine kinases with postsynaptic densities after transient cerebral ischemia in the rat brain. J Cereb Blood Flow Metab 2000, 20:505-512

43. Tian G, Cory M, Smith AA, Knight WB: Structural determinants for potent selective dual site inhibition of human pp60c-src by 4-anilinoquinazolines. Biochemistry 2001, 40:7084-7091

44. Yang M, Leonard JP: Identification of mouse NMDA receptor subunit NR2A C-terminal tyrosine sites phosphorylated by coexpression with v-Src. J Neurochem 2001, 77:580-588

45. Everall I, Vaida F, Khanlou N, Lazzaretto D, Achim C, Letendre S, 
Moore D, Ellis R, Cherne M, Gelman B, Morgello S, Singer E, Grant I, Masliah E: Cliniconeuropathologic correlates of human immunodeficiency virus in the era of antiretroviral therapy. J Neurovirol 2009, $1-11$

46. Chen B-S, Roche KW: Regulation of NMDA receptors by phosphorylation. Neuropharmacology 2007, 53:362-368

47. Shamloo M, Wieloch T: Changes in protein tyrosine phosphorylation in the rat brain after cerebral ischemia in a model of ischemic tolerance. J Cereb Blood Flow Metab 1999, 19:173-183

48. Dunah AW, Wang Y, Yasuda RP, Kameyama K, Huganir RL, Wolfe BB, Standaert DG: Alterations in subunit expression. composition, and phosphorylation of striatal $\mathrm{N}$-methyl-D-aspartate glutamate receptors in a rat 6-hydroxydopamine model of Parkinson's disease. Mol Pharmacol 2000, 57:342-352

49. Sze C-I, Bi H, Kleinschmidt-DeMasters BK, Filley CM, Martin LJ: $\mathrm{N}$-Methyl-D-aspartate receptor subunit proteins and their phosphorylation status are altered selectively in Alzheimer's disease. J Neurol Sci 2001, 182:151-159

50. Grosshans DR, Clayton DA, Coultrap SJ, Browning MD: LTP leads to rapid surface expression of NMDA but not AMPA receptors in adult rat CA1. Nature Neurosci 2002, 5:27-33

51. Zheng F, Gingrich MB, Traynelis SF, Conn PJ: Tyrosine kinase potentiates NMDA receptor currents by reducing tonic zinc inhibition. Nature Neurosci 1998, 1:185-191

52. Iwamoto T, Yamada Y, Hori K, Watanabe $\mathrm{Y}$, Sobue K, Inui M: Differential modulation of NR1-NR2A and NR1-NR2B subtypes of NMDA receptor by PDZ domain-containing proteins. J Neurochem 2004, 89:100-108

53. Kalia LV, Gingrich JR, Salter MW: Src in synaptic transmission and plasticity. Oncogene 2004, 23:8007-8016

54. Yamada Y, Iwamoto T, Watanabe Y, Sobue K, Inui M: PSD-95 eliminates Src-induced potentiation of NR1/NR2A-subtype NMDA recep- tor channels and reduces high-affinity zinc inhibition. J Neurochem 2002, 81:758-764

55. Seabold GK, Burette A, Lim IA, Weinberg RJ, Hell JW: Interaction of the tyrosine kinase Pyk2 with the N-methyl-D-aspartate receptor complex via the Src homology 3 domains of PSD-95 and SAP102. J Biol Chem 2003, 278:15040-15048

56. Haughey NJ, Nath A, Mattson MP, Slevin JT, Geiger JD: HIV-1 Tat through phosphorylation of NMDA receptors potentiates glutamate excitotoxicity. J Neurochem 2001, 78:457-467

57. Cull-Candy SG, Leszkiewicz DN: Role of distinct NMDA receptor subtypes at central synapses. Sci STKE 2004, 2004:re16, 1-9

58. Cull-Candy S, Brickley S, Farrant M: NMDA receptor subunits: diversity, development and disease. Curr Opin Neurobiol 2001, 11:327335

59. Eugenin EA, King JE, Hazleton JE, Major EO, Bennett MV, Zukin RS, Berman JW: Differences in NMDA receptor expression during human development determine the response of neurons to HIV-Tat-mediated neurotoxicity. Neurotox Res 2010, DOI: 10.100715/2640-010-9150-X

60. Kalia LV, Kalia SK, Salter MW: NMDA receptors in clinical neurology: excitatory times ahead. Lancet 2008, 7:742-755

61. Schifitto G, Navia BA, Yiannoutsos CT, Marra CM, Chang L, Ernst T, Jarvik JG, Miller EN, Singer, EJ, Ellis RJ, Kolson DL, Simpson D, Nath A, Berger J, Shriver SL, Millar LL, Colquhoun D, Lenkinski R, Gonzalez RG, Lipton SA; Adult AIDS Clinical Trial Group (ACTG) 301; 700 Teams; HIV MRS Consortium: Memantine and HIV-associated cognitive impairment: a neuropsychological and proton magnetic resonance spectroscopy study. AIDS 2007, 21:1877-1886

62. Schifitto G, Yiannoutsos CT, Simpson DM, Marra CM, Singer EJ, Kolson DL, Nath A, Berger JR, Navia B, Team AACTG: A placebocontrolled study of memantine for the treatment of human immunodeficiency viruses associated sensory neuropathy. J Neurovirol 2006, 12:328-331 